\title{
Les théories des mouvements sociaux et la dialectique des niveaux : un cadre d'analyse pour l'étude des évolutions d'Al-Qaïda au Maghreb islamique
}

\author{
Adib Bencherif \\ Université du Québec à Montréal, Chercheur en résidence à l'Observatoire sur le Moyen-Orient et \\ l'Afrique du Nord de la Chaire Raoul-Dandurand
}

Les théories des mouvements sociaux sont de plus en plus utilisées pour étudier les groupes terroristes. Elles comportent différents niveaux d'analyse: les niveaux macro-analytique, méso-analytique et micro-analytique. Le présent article tente d'adapter ce corpus théorique à l'étude du groupe jihadiste Al-Qaïda au Maghreb islamique (AQMI). En effet, au cours des dernières années, AQMI ne s'est pas développé au Maghreb alors que ses activités ont augmenté exponentiellement dans la région sahélienne, au point d'y développer un sanctuaire pour le groupe au nord du Mali. Le développement d'AQMI au Sahel est-il alors le résultat de choix stratégiques ou celui de dynamiques internes? Pour expliquer le développement d'AQMI dans la région sahélo-saharienne, l'auteur propose une grille de lecture basée sur les niveaux macro et méso-analytiques et sur leur mise en dialectique. Le niveau macro-analytique met en lumière la structure des opportunités politiques induisant les choix stratégiques d'AQMI et le niveau méso-analytique les dynamiques internes du groupe. La littérature existante sur AQMI étant principalement constituée de monographies et de notes de recherches, l'ambition de cet article est alors de concilier théorie et recherche empirique. Ainsi le cadre d'analyse proposé est inspiré des approches de l'action collective et de la mobilisation des ressources et cherche à améliorer la compréhension des évolutions du groupe. L'étude menée est une analyse qualitative centrée sur les États algérien et malien.

Mots clés : terrorisme ; Al-Qaïda au Maghreb islamique; AQMI ; mouvements sociaux

The theories of social movements are increasingly used to study terrorist groups and they are characterized by three different levels of analysis: macro, meso, and micro. This article attempts to adapt this theoretical framework to the study of the jihadist group al-Qaeda in the Islamic Maghreb (AQIM). Indeed, in recent years, AQIM failed to develop in North Africa while its activities have increased exponentially in the Sahel region, to the point of developing a sanctuary for the group in northern Mali. Is the development of AQIM in the Sahel the result of strategic choices or internal dynamics? To explain the development of AQIM in the Sahel-Saharan region, the author proposes a reading grid based on the macro and meso levels of analysis and their dialectical implementation. The macro-level analysis highlights the political opportunities structure inducing AQIM strategic choices and the meso-level analysis focuses on internal dynamics of the group. Seeing as existing literature on AQIM is mainly constituted of monographs and research notes, the intent of this paper is then to reconcile theory and empirical research. Thus, the proposed framework is inspired from collective action and resource mobilization approaches and seeks to improve the understanding of the evolution of the group. The study is a qualitative analysis focusing on the Algerian and Malian States.

Keywords : terrorism ; al-Qaeda in the Islamic Maghreb ; AQIM ; social movements 
Au cours des dernières années, Al-Qaïda au Maghreb islamique (AQMI) s'est considérablement développée dans la région sahélo-saharienne et a progressivement établi son sanctuaire au nord du Mali. Le groupe a ainsi su tisser des relations avec les réseaux criminels et participer aux trafics de drogue sillonnant la région. Par ailleurs, l'implication du groupe dans l'insurrection au nord du Mali, amorcée en janvier 2012, a révélé qu'il pouvait constituer une réelle menace à la stabilité des États sahéliens en se mêlant aux conflits latents et aux tensions sociales existantes. Pourtant, originellement, et comme l'indique son nom, le théâtre opérationnel d'AQMI est le Maghreb.

En effet, AQMI est issue de l'évolution du Groupe salafiste pour la prédication et le combat (GSPC), un groupe jihadiste algérien qui, au nom de sa survie, s'est recyclé dans le jihad global. Pour ce faire, le GSPC a intégré, suite à un certain nombre de transformations, Al-Qaïda le 11 septembre 2006 et a changé son nom pour AQMI le 24 janvier 2007. Cependant, malgré le label d' "Al-Qaïda», AQMI est un groupe sui generis et conserve des particularités qui lui sont propres. AQMI évolue ainsi constamment en fonction des contraintes et des opportunités de son environnement régional. Ce sont ces dernières qui l'ont a priori conduit à se développer au Sahel. Néanmoins, ce développement s'accompagne de tensions et de rivalités entre les émirs du groupe. Cette étude tente donc d'examiner si le développement d'AQMI dans la région sahélo-saharienne est le fruit de confrontations et de rapports de force au sein du groupe ou de choix stratégiques du groupe en réponse à des contraintes et opportunités environnementales. Considérant la violence politique comme un processus, l'hypothèse avancée est que le développement d'AQMI dans l'espace sahélo-saharien est le résultat de deux dynamiques : les contraintes et les opportunités offertes par l'environnement régional dans un premier temps et les dynamiques internes du groupe sous-tendant un jeu de pouvoir, d'influence et des compromis entre les cadres et émirs du groupe, dans un second temps. Certaines décisions du groupe seraient donc prises collégialement, AQMI s'apparentant à un acteur unitaire rationnel. D'autres, résultant des rapports de force et des rivalités entre les cadres et émirs, conduisent à considérer le groupe comme doté d'une certaine rationalité, laquelle serait fragmentée entre les différents décideurs du groupe qui défendent prioritairement leurs propres intérêts. ${ }^{1}$

Dans un premier temps, nous soulignerons, par une revue de la littérature existante, l'apport conceptuel et analytique des théories des mouvements sociaux. En effet, les approches portant sur les opportunités politiques et sur la mobilisation des ressources permettent de mieux appréhender les groupes terroristes. Se dégageront alors, dans un second temps, deux niveaux d'analyse : les niveaux macroanalytique et méso-analytique. L'opérationnalisation du rapport dialectique entre ces niveaux apportera une compréhension plus dynamique et structurée à l'étude de ce groupe. Enfin, il s'agira d'appliquer cette grille de lecture à notre étude de cas qui s'étend de la création du GSPC en Algérie en 1998 jusqu'aux récentes activités d'AQMI au nord du Mali avant l'intervention française en janvier 2013. Le développement d'AQMI dans l'espace sahélo-saharien étant le résultat observé de l'évolution du groupe, la mobilisation de ce corpus théorique permet d'identifier plus nettement les causes endogènes et exogènes au groupe, de les mettre en relation et de les confronter à la réalité des faits.

\section{Apports conceptuels et analytiques des théories des mouvements sociaux}

Les théories des mouvements sociaux subsument différentes approches, notamment celles des opportunités politiques, des structures de mobilisation et des processus de cadrage (Revillard, 2003). Néanmoins, ces théories ne se sont en vérité intéressées à l'étude de l'émergence et du développement des groupes terroristes que relativement récemment (Rapin, 2008, p.195). Les difficultés notamment heuristiques liées à l'étude du terrorisme (Sidel, 2006, p.220), phénomène pluriel n'ayant pas de définition consensuelle au sein de la littérature académique (Duyvesteyn, 2004), ont probablement contribué à ralentir le processus d'intégration des théories des mouvements sociaux aux études des groupes terroristes.

\footnotetext{
${ }^{1}$ Ces dynamiques s'apparentent aux mécanismes bureaucratiques décrits par Graham Allison. Allison, G.T. (1969). Conceptual Models and the Cuban Missile Crisis. American Political Science Review, 63(3), 689-718.
} 
Toutefois, au cours des deux dernières décennies, de nombreux travaux ont vu le jour. De rigoureux cadres d'analyse sur plusieurs niveaux ont ainsi été élaborés pour étudier les groupes terroristes (Della Porta, 1995). Néanmoins, les études mettant en relation les différents niveaux d'analyse, soit les niveaux macro-analytique, méso-analytique et micro-analytique, se font encore rares (Bosi, 2012, p.188). En effet, la majeure partie des études réalisées sur la violence politique se focalisent sur un des niveaux en négligeant les autres, conduisant de ce fait à présenter des explications partielles et souvent insuffisantes.

Ainsi, les tenants du niveau macro-analytique se concentrent sur l'environnement dans lequel va émerger le groupe, c'est-à-dire les contraintes et opportunités externes aux mouvements. Ces dernières sont conceptualisées à travers la notion de structure des opportunités politiques (Rapin, 2008, p.196). La structure des opportunités politiques correspond à la structure des relations de pouvoir, à la fois institutionnelles et informelles, figurant au sein d'un système politique national (McAdam, McCarthy \& Zald, 1996/1997, p.3). Les changements de configuration de la structure des opportunités politiques influent donc sur les choix stratégiques réalisés par le mouvement (McAdam et al, 1996/1997, p.12; Della Porta, 2012, p.6). Par ailleurs, les analyses réalisées sur les mouvements sociaux sont généralement stato-centrés (Della Porta, 2008, p.224).

Cependant, dans le cadre d'étude de groupes islamistes violents, de groupes jihadistes s'inscrivant dans un jihad global, ou plutôt « glocal» dans le cas d'AQMI (Marret, 2008), il convient de dépasser cette grille de lecture. En effet, AQMI est un groupe « glocal » évoluant en fonction des opportunités et des contraintes locales tout en revendiquant un jihad global. Dès lors, la structure des opportunités politiques d'ordre international se croise et s'entremêle avec la structure des opportunités politiques nationales (Della Porta, 2008, pp.224-225). En effet, la lutte contre de pareils groupes conduit à une coopération entre États. Les groupes sont dès lors soumis à des contraintes nationales et internationales. Cela est d'autant plus pertinent lorsque les groupes se développent dans des régions telles que celles du Sahara et du Sahel où la réalité sédentaire de la séparation par des frontières s'efface au moins partiellement pour se conjuguer à la réalité nomade (Retaillé \& Walter, 2011). Des dimensions culturelles et sociales s'ajoutent d'ailleurs à la dimension politique. Ainsi, la considération de l'environnement dans lequel se développe le groupe à travers la notion de structure des opportunités politiques, ne recouvrant que l'unique dimension politique, peut s'avérer incomplète même si cette dimension demeure probablement souvent dominante (Rucht, 1996/1997). Le concept de contexte structurel a alors été avancé dans la littérature pour recouvrir à la fois les dimensions politique, sociale et culturelle (Rucht, 1996/1997). Ce concept nous apparait plus pertinent dans la présente étude. L'étude d'un groupe tel qu'Al-Qaïda au Maghreb islamique oblige à une flexibilité dans la grille de lecture en raison de sa réalité mouvante, évolutive et transnationale. De plus, l'objectif politique du groupe à court et moyen terme étant, outre le jihad global revendiqué, la survie, il conduit nécessairement à des prises de décision pragmatiques de la part des émirs et cadres du groupe (Pham, 2011). Ces derniers cherchent à s'adapter à leur environnement régional au nom de la survie d'AQMI en répondant aux différentes politiques des États de la région tout en essayant de se greffer aux réalités sociales et culturelles sahélo-sahariennes. Cela conforte la nécessité de considérer les contraintes et opportunités à travers les différentes dimensions et en dépassant le cadre d'une analyse stato-centrée.

Les tenants du niveau méso-analytique se sont, quant à eux, essentiellement focalisés sur les dynamiques internes des mouvements sociaux. Deux écoles de pensée se sont développées et confrontées, la première priorisant l'étude des structures organisationnelles formelles des mouvements et la seconde portant son attention sur les structures informelles. Ainsi, les partisans de la théorie de la mobilisation des ressources décrivent les organisations de mouvements sociaux dans un schème proche des organisations formelles (McAdam et al, 1996/1997; Wiktorowicz, 2004, pp.9-13; Beck, 2008, pp.1568-1569). En effet, la théorie de la mobilisation des ressources considère les mouvements sociaux comme des décideurs collectifs rationnels définissant et appliquant les stratégies les plus utiles au groupe en fonction des ressources matérielles et humaines disponibles (Kitschelt, 1986, p.59). Les partisans du modèle du processus politique se sont davantage penchés sur les structures informelles et les réseaux sociaux jouant un rôle conséquent dans l'émergence et le développement des groupes (MacAdam et al, 1996/1997; Wiktorowicz, 2004, pp.9-13). McAdam (1996) qualifie ces structures informelles de « contextes de micromobilisation ». 
Dans la présente étude, la théorie de la mobilisation des ressources sera celle utilisée au niveau méso-analytique pour mettre l'emphase sur la structure organisationnelle du groupe et sur les relations de pouvoir en son sein. L'importance des réseaux sociaux et des structures informelles n'est pas à négliger, mais ne correspond pas à l'angle adopté dans notre analyse.

Enfin, le niveau micro-analytique s'intéresse davantage aux perceptions et aux valeurs partagées par les militants du groupe à travers les processus de cadrage. Le concept de cadrage pourrait se résumer aux « efforts stratégiques conscients déployés par des groupes de personnes pour façonner des compréhensions communes du monde et d'eux-mêmes qui légitiment et motivent l'action collective » (Revillard, 2003, p.12; McAdam et al, 1996/1997, p.6; Snow, Rochford, Worden \& Benford, 1986). Ainsi, le niveau micro-analytique, plus idéel que structurel, contrairement aux deux autres niveaux, porte son attention sur les motifs des individus et sur les processus cognitifs et culturels déterminant leurs interprétations des situations et évènements vécus. (McAdam et al, 1996/1997, pp.5-7; Rapin, 2008, p.196). Bien qu'il soit complémentaire des deux autres niveaux, et sans remettre en question sa pertinence, le niveau micro-analytique ne sera pas mobilisé dans la présente étude, le groupe étudié étant un groupe déjà constitué s'inscrivant dans une dynamique de survie et donc dans un agir nécessairement pragmatique. ${ }^{2}$ Par ailleurs, les décideurs d'AQMI sont appelés à effectuer des calculs coûts-bénéfices pour pouvoir permettre au groupe de survivre. Notre démarche se fait alors résolument rationaliste et se focalise sur les niveaux macroanalytique et méso-analytique pour expliquer ainsi, par les dynamiques existantes entre l'environnement et le groupe, le développement d'AQMI au Sahel. Pour autant, la pertinence du niveau micro-analytique n'est pas remise en question et une dialectique entre ce niveau et les autres s'avère nécessaire pour appréhender la réalité d'un groupe dans sa globalité (Della Porta, 1995). Une étude plus ambitieuse d'AQMI pourrait d'ailleurs compléter celle-ci et le prendre en compte, éclairant probablement avec davantage d'acuité les évolutions du groupe. Nous supposons toutefois que l'intégration du niveau micro-analytique à l'étude du groupe complètera plus qu'il ne contredira les conclusions obtenues par l'analyse des deux autres niveaux.

\section{Les niveaux macro-analytique et méso-analytique : opérationnalisation et dialectique}

L'une des faiblesses figurant dans la littérature est l'absence de dialectique entre niveaux d'analyse (Bosi, 2012, p.188). Certains travaux peuvent s'intéresser à différents niveaux, mais ont pour défaut de ne pas suffisamment les mettre en relation. Dans la présente étude, nous tentons l'opérationnalisation de la dialectique des niveaux macro- et méso-analytique en nous inspirant des travaux de Rucht (1996/1997). En premier lieu, le niveau macro-analytique a été précédemment défini comme étant le contexte structurel. Le groupe opérant dans différents États, il sera étudié à travers différents contextes structurels nationaux. En effet, les groupes jihadistes tels qu'AQMI n'évoluent pas dans un seul État. Pour la présente étude, les contextes structurels algérien et malien seront ceux sur lesquels l'analyse se centrera. Sans prétendre à l'exhaustivité des territoires où le groupe opère, ces deux États ont été choisis, car AQMI est originellement une organisation terroriste algérienne s'étant internationalisée et ayant in fine développée son sanctuaire sahélien au nord du Mali.

Le contexte structurel est donc considéré à travers trois dimensions: les dimensions politique, sociale et culturelle. La dimension politique est définie, suivant les travaux de Rucht (1996/1997, p.190), à travers les quatre facteurs suivants : l'accès aux décisions politiques, la capacité d'implantation des politiques, la configuration des alliances et la configuration conflictuelle. Dans le cadre d'un groupe tel qu'AQMI, l'accès aux décisions politiques au sens de participation dans une configuration institutionnelle se révèle très peu pertinent, car le groupe est internationalement reconnu comme terroriste. Avoir accès formellement aux appareils politiques d'un État est hautement improbable pour un pareil groupe. Toutefois, si l'on ne considère ce facteur qu'à travers les canaux informels, l'ensemble des variables peut s'avérer adapté pour expliquer le processus de développement du groupe jihadiste.

Le facteur de la capacité de l'implantation des politiques revient quant à lui à s'interroger sur la capacité de l'État à mettre en œuvre des politiques publiques sans résistances interne ou externe. La

\footnotetext{
${ }^{2} \mathrm{Ce}$ choix s'avère aussi nécessaire par manque de sources.
} 
structure des alliances se questionne sur la présence ou non d'alliés du groupe au sein du régime et des appareils étatiques. Finalement, le facteur de la configuration conflictuelle s'intéresse à la capacité de répression de l'État contre le groupe (Rucht, 1996/1997, pp.190-192). Ce dernier facteur est évidemment l'un des plus souvent soulevés par la littérature (Della Porta, 2008).

Par ailleurs, la dimension sociale se penche sur le milieu social, sur les possibilités de socialisation des activistes avec la population (Rucht, 1996-1997) et sur leurs capacités à intégrer les réseaux sociaux existants. Enfin la dimension culturelle se résumera dans la présente étude à s'intéresser aux valeurs portées par la société et à leur compatibilité avec les valeurs du groupe (Rucht, 1996/1997).

En second lieu, le niveau méso-analytique se penche sur les dynamiques internes au groupe. Elles se subdivisent en deux variables : la structure du groupe et les relations de pouvoir entre les décideurs du groupe. Ces deux variables peuvent donner lieu à des mécanismes sociaux pouvant créer entre autres un enfermement du groupe sur lui-même dans la radicalisation d'une position (Della Porta, 1995) ou à des tensions pouvant aboutir en toute fin à des dissidences.

Notant que le groupe obéit à une dynamique de survie et que notre étude ne se focalise pas sur son émergence, mais son développement, notre dialectique partira du niveau macro-analytique. En effet, un contexte structurel favorable peut conduire au développement du groupe. Par contre un contexte structurel défavorable peut amener un groupe islamiste s'inscrivant dans le jihad global à diminuer au moins temporairement ses activités ou le conduire à se développer dans un autre État que l'État d'origine. La structure organisationnelle du groupe peut donc se centrer sur un État, ou un ensemble d'États, s'y développer, et modifier alors les relations de pouvoir entre décideurs du groupe. La hiérarchie du groupe préalablement définie est alors remise en question, polarisant dès lors les positions des émirs du groupe, favorisant le développement d'agendas politiques personnels et rendant très probables les tensions au sein du groupe.

Par ailleurs, s'il y a perte de la réalité verticale et hiérarchique de l'organisation, de nouveaux équilibres dans les rapports de force au sein du groupe peuvent être aussi favorisés. Ainsi, le contexte structurel peut agir sur les rapports de force au sein du groupe, mais aussi sur la structure organisationnelle. Les rapports de force et la structure organisationnelle s'influencent aussi entre eux, renforçant les éventuelles transformations. L'aplanissement et le relâchement acquis par le groupe par exemple, peuvent le conduire à s'établir en un réseau fait d'un ensemble de structures de mobilisation autonomes (Della Porta, 2008, p.226).

Dès lors, la restructuration du groupe autour des ressources disponibles peut influer sur le contexte structurel par une augmentation ou une diminution de ses activités. L'augmentation des activités du groupe dans une région peut diminuer par exemple la capacité d'implantation des politiques de l'État dans certaines régions et renforcer la configuration conflictuelle. La reconfiguration du contexte structurel peut alors conduire à de nouvelles contraintes et opportunités exercées sur le groupe, entraînant du même coup de nouvelles interactions au sein du groupe et de nouvelles réponses.

Les contextes structurels conduisent ainsi à favoriser ou non l'adoption de certaines structures organisationnelles. Ces structures conduisent à revoir les rapports de force au sein du groupe et engendrent des dynamiques internes pouvant favoriser parfois une prise de décision du groupe dans une logique de choix stratégique collectif consensuel ou au contraire donner lieu à des tensions et discordes permettant parfois au groupe de se développer, mais sans réelle entente en amont. Les réponses adoptées par le groupe peuvent alors conduire à une évolution des contextes structurels qui eux-mêmes par les réponses apportées par l'État et la société, suscitent de nouvelles dynamiques internes. C'est cette dialectique que l'on tentera de mettre en œuvre dans l'analyse du groupe AQMI. Il convient de noter aussi que l'existence de tensions et de discordes récurrentes causées par des changements environnementaux et les dynamiques internes peut entraîner des dissidences. 


\section{Schéma résumant les principales dynamiques entre le contexte structurel et le groupe}

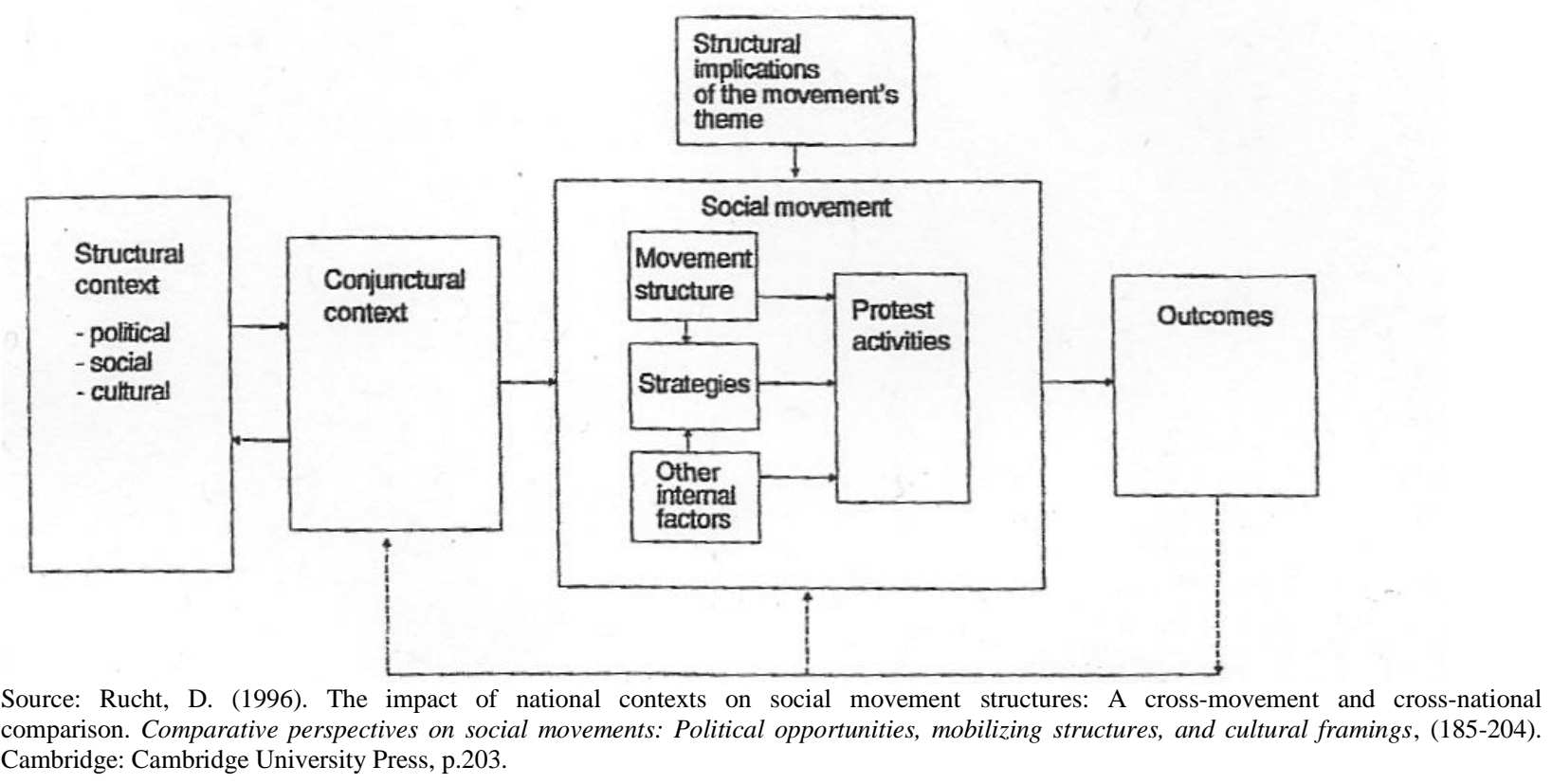

\section{L'application de la grille de lecture à l'étude d'AQMI}

En 1998, le Groupe salafiste pour la prédication et le combat (GSPC) est créé. Reliquat de la guerre civile algérienne des années 1990, il est constitué suite à la défection d'anciens membres du Groupe islamique armé (GIA). Les leaders du GSPC, dont l'émir national Hassan Hattab, cherchent à redorer l'image du jihad en Algérie en se dissociant du GIA ayant perpétré des massacres au sein de la population (Filiu, 2011).

Néanmoins, le contexte structurel est très peu favorable à la survie et au maintien du GSPC. En effet, dès l'entrée en fonction du président algérien Abdelaziz Bouteflika en 1999, une politique de réconciliation nationale est mise en œuvre pour amnistier les islamistes décidant de rendre les armes. Une pareille décision politique implique une très forte capacité d'implantation des politiques de l'État algérien et de ses représentants. Le président Bouteflika, arrivant en homme providentiel suite à la guerre civile et n'ayant pas exercé le pouvoir au cours des deux dernières décennies, bénéficie donc, lors de son arrivée au pouvoir, d'un capital de confiance auprès de l'armée et de la société. Des milliers de jihadistes se rendront, affaiblissant de ce fait considérablement les derniers groupes jihadistes et le GSPC qui cherche à s'établir. Par ailleurs, une véritable guerre continue d'être livrée aux groupes jihadistes ne se rendant pas. Suite aux opérations de ratissage, menées dans les régions montagneuses kabyles, où se concentrent les principales forces du front nord du GSPC, le groupe se voit menacer de disparaître (Durand, 2011). La configuration conflictuelle ne permet donc pas au GSPC de se régénérer et ne cesse de l'affaiblir. Même parmi les islamistes, dont ceux ayant participé à la guerre civile tel que le Front islamique du Salut (FIS), le GSPC ne bénéficie d'aucun allié politique et n'a accès à aucun canal informel pour influer sur les décisions politiques.

De plus, les dimensions sociales et culturelles sont aussi défavorables au maintien et au développement du groupe en Algérie que les dimensions politiques. Le GIA, par les attaques répétées contre la population algérienne, a terni l'image des islamistes en général, et a fortiori des jihadistes (Durand, 2011). Les valeurs de la société deviennent par là même incompatibles avec celles des groupes jihadistes, et donc avec celles du GSPC.

La marginalisation progressive du groupe semble le condamner à l'extinction et oblige les cadres et émirs à en repenser les objectifs stratégiques. Après les attentats du 11 septembre 2001 et la mise en avant du jihad global par l'organisation terroriste Al-Qaïda, les dynamiques internes du groupe, au niveau 
donc méso-analytique, se résument en une polarisation en deux ailes défendant deux visions différentes de l'avenir du GSPC : l'aile des internationalistes et celle des « algérianistes » (Botha, 2008; Durand, 2011; Lounnas 2011). L'aile des internationalistes défend l'idée d'étendre le combat et de basculer dans le jihad global. L'aile des « algérianistes » préfère par contre continuer à mener le jihad exclusivement en Algérie (Botha, 2008 ; Durand, 2011; Lounnas, 2011).

La guerre de 2003 en Irak va marquer un tournant dans l'histoire du groupe et favoriser la vision défendue par les internationalistes. Le groupe s'inscrivant dans une dynamique de survie, la divergence se fait donc davantage sur le plan stratégique qu'idéologique. La stratégie la plus pertinente, au vu des contextes structurels international et algérien, se révèle être celle mettant en avant le jihad global. Il s'agit d'une reconversion dans le combat pour relancer le groupe et d'un aveu tacite de l'échec du combat à une échelle strictement nationale (Botha, 2008). En effet, en invoquant une rhétorique anti-impérialiste et antioccidentale, en changeant dès lors d'ennemis, priorisant, au moins dans le discours, « l'ennemi lointain sur l'ennemi proche », le groupe cherche à acquérir de nouvelles ressources (Lounnas, 2011, 2012). Se régénérant en recrutant dans une nouvelle génération sensible au salafisme et à l'anti-impérialisme, le GSPC parvient à regarnir ses rangs (Lounnas, 2012). Les émirs du GSPC promettent alors aux jeunes jihadistes nouvellement recrutés, venant d'Algérie, mais aussi de l'ensemble des pays du Maghreb, de les envoyer mener le jihad en Irak après leur entraînement (Durand, 2011).

Les contraintes incarnées par les politiques algériennes et l'armée algérienne, ainsi que l'opportunité de s'inscrire dans le jihad global à la suite de la guerre en Irak de 2003, engendrent donc des dynamiques internes au sein du groupe. Au niveau méso-analytique, un nouvel équilibre dans les rapports de force au sein du GSPC se dessine, les internationalistes imposant leurs visions et prenant possession des rênes du pouvoir. L'arrivée d'une nouvelle génération sensibilisée au jihad global facilite ces nouveaux rapports de force au sein du groupe. En effet, l'émir du GSPC, Hassan Hattab, est destitué et Nabil Sahrawi, appartenant à l'aile internationaliste, est nommé émir national en septembre 2003 (Lounnas, 2012).

Cependant, c'est à la mort de ce dernier et avec l'arrivée d'Abdelmalek Droukdal à la tête du groupe en juin 2004 que l'internationalisation du GSPC s'accélère et qu'un réel rapprochement avec Al-Qaïda s'opère (Durand, 2011). Droukdal va très vite établir une coopération régulière entre son groupe et $\mathrm{Al}$ Qaïda en Irak (AQI). Mobilisant les ressources du groupe dans une dynamique internationale par l'envoi de jeunes jihadistes dans le théâtre irakien, il cherche à devenir l'organisation jihadiste pivot de la région et à augmenter les ressources du GSPC par la cooptation d'autres groupes jihadistes et le recrutement de nouveaux membres (Guidère, 2008 ; Filiu, 2009, 2011). Simultanément, l'objectif visé par Droukdal est de se rapprocher des leaders d'Al-Qaïda par l'intermédiaire d'Abou Moussab Zarkawi, leader d'AQI, pour pouvoir intégrer l'organisation Al-Qaïda. Al-Zawahiri, alors numéro deux d'Al-Qaïda, finit par accepter d'examiner le rattachement du GSPC, mais après une période de probation d'un an, allant de septembre 2005 à 2006. Le GSPC effectue alors un certain nombre de changements, notamment dans la structure organisationnelle, pour pouvoir intégrer Al-Qaïda (Filiu, 2009), renforçant dès lors les tensions entre « algérianistes » et internationalistes au sein du groupe.

Intégrant des membres d'autres groupes présents en Afrique du Nord, tels que le Groupe islamique des combattants libyens (GICL), ainsi que des groupes de jihadistes marocains et tunisiens, mais aussi des groupuscules sahélo-sahariens, le groupe étend son théâtre d'opérations à l'ensemble du Maghreb (Guidère, 2008; Daniel, 2012). La structure de commandement reste toutefois dominée par les jihadistes algériens du GSPC (Daniel, 2012). Néanmoins, les « algérianistes » du groupe considèrent que la mission originelle est dénaturée et que le groupe se doit de prioriser le jihad en Algérie. Malgré les tentatives de conciliation et de compromis tentées par l'émir Droukdal, cherchant à les ménager en promettant de continuer le jihad en Algérie, le groupe subit de nombreuses défections, conséquence du tournant stratégique et idéologique (Guidère, 2008). Les contextes structurels ont ainsi modifié un certain nombre de dynamiques internes au sein du groupe. Des changements de rapports de force au profit des internationalistes et des transformations de la structure organisationnelle du groupe ont été dès lors réalisés, entraînant simultanément des défections.

Le 11 septembre 2006, Al-Zawahiri annonce officiellement l'intégration du groupe au sein d'AlQaïda. Le processus est finalisé le 24 janvier 2007, jour du changement d'appellation du groupe. Le GSPC 
devient «Al-Qaïda au Maghreb islamique » (Lounnas, 2012). Pour autant, même après l'adoption de ce nom, le groupe reste essentiellement centré en Algérie et ne réalise que quelques attentats dans les autres pays du Maghreb. AQMI ne parvient pas à s'y implanter et continue de s'affaiblir suite aux opérations menées en Algérie (Porter, 2011). Les contextes structurels marocain, tunisien et algérien ne permettent pas l'essor du groupe. Les politiques et les armées de ces pays se révèlent efficaces pour lutter contre le groupe. Quant au soutien des populations, AQMI n'est pas parvenue à l'obtenir. Le front nord d'AQMI, essentiellement résumé au Nord-Est du territoire algérien, est alors acculé et ne peut se développer au vu des contextes structurels des États du Maghreb (Porter, 2011).

C'est ainsi que le front sud va progressivement être l'alternative priorisée pour la survie du groupe et conduire à son développement dans la région sahélo-saharienne. Dès les années 1990 avec le GIA, les brigades du groupe, composées de centaines d'hommes armés et appelés katibas, écument le désert du Sahara et participent aux différents trafics de la région sahélo-saharienne (Daniel, 2012; Lounnas 2012). La katiba la plus active est celle de Mokhtar Belmokhtar. Ce dernier a su au fil du temps tisser d'importants réseaux au sein des différentes tribus touarègues et arabes de la région sahélo-saharienne, notamment au nord du Mali (Daniel, 2012; Lounnas 2012).

En effet, l'échec du développement économique du territoire nord-malien et l'intégration non aboutie des populations touarègues au sein de l'appareil étatique malien après la décolonisation a conduit certains des membres des tribus de la sous-région à intégrer des réseaux de contrebande (Smith, 2009). Belmokhtar a donc su profiter des réseaux sociaux existants, mais cela n'aurait pas été permis si le contexte structurel malien n'avait pas été favorable au développement de ses activités (International Crisis Group [ICG], 2012).

L'État malien, ayant opté pour un contrôle à faible coût et à distance du territoire nord-malien pour éviter d'éventuels affrontements ou tensions avec les populations touarègues, la zone y est peu militarisée (ICG, 2012). La capacité d'implantation de politiques est aussi très faible et délicate. La configuration conflictuelle est donc quasi-absente, car l'État malien s'inscrit plus dans un laissez-faire tant que n'existe pas de troubles sur son territoire. En échange de l'établissement du sanctuaire du groupe au nord du Mali, une entente tacite semble alors avoir été développée entre AQMI et les autorités. En effet, comparativement à la Mauritanie voisine, à l'Algérie ou au Niger, le Mali n'a que très peu été la victime d'attentats et d'attaques par AQMI au cours de la dernière décennie. Les membres d'AQMI semblent s'être engagés à ne pas perpétrer d'attentats ou réaliser d'attaques sur le territoire malien (Filiu, 2010). Le contexte structurel malien a donc été favorable au développement d'AQMI dans la région septentrionale du pays.

Par ailleurs, les populations ne se sont pas montrées ouvertement hostiles à ce groupe au nord du Mali. Belmokhtar a su aussi garder un profil bas tout en s'alliant à des membres des différentes tribus et ethnies de la région (Lounnas, 2012). Il a su dès lors s'intégrer et participer aux différents trafics de cigarettes, de drogues et d'armes de la région sahélo-saharienne (Walther \& Retaille, 2011). Ces trafics incarnent la principale source de financement du GIA, puis du GSPC et enfin d'AQMI dans ses premières années. Les ressources financières du groupe semblent provenir donc essentiellement du front sud et de la katiba de Belmokhtar jusqu'en 2008.

L'émir Droukdal et les katibas dans le Nord-Est algérien entretiennent donc une relation de dépendance avec les katibas du Sahel pour la fourniture d'armes et d'équipements. Dès son intronisation à la tête du groupe, Droukdal semble chercher à affaiblir le très influent Belmokhtar qu'il considère comme un rival capable de le renverser. Pour ce faire, il nomme notamment Abou Zaïd émir d'une katiba concurrente au Sahel. S'il ne peut se permettre de s'en séparer, au vu de l'importance de Belmokhtar pour le financement d'AQMI, il ne cessera de chercher à le marginaliser au sein du groupe. C'est ainsi qu'en passant par les organes de décision du groupe, c'est-à-dire le Conseil des chefs et le Conseil consultatif, Droukdal et son entourage ôtent le titre d' « émir du Sahara » à Belmokhtar en 2005 (Lounnas, 2012) et nomme dans la région sahélo-saharienne un coordonnateur, officiellement le numéro deux du groupe pour superviser l'ensemble des katibas du front sud.

En réponse à la nécessité de développer le front sud, seule option viable puisqu'AQMI ne parvient pas à se développer au Maghreb et a besoin de ressources matérielles, le groupe se restructure, mais sur fond de tensions. Droukdal ne veut pas voir les relations de pouvoir s'inverser et cherche à conserver un 
contrôle sur les émirs des katibas sahéliennes. Néanmoins, le contexte structurel algérien isole de plus en plus Droukdal et le front nord, alors que le contexte structurel malien permet le développement des katibas du front sud.

Bien qu'AQMI soit constituée autour d'une organisation ayant deux organes de décision, le Conseil des chefs et le Conseil consultatif, et plusieurs comités tels que les comités juridique, militaire et médiatique (Africa Center for the Study and Research on Terrorism [CAERT], 2010), la structure organisationnelle du groupe n'est pas extrêmement hiérarchisée et tend même à s'aplanir à travers le nécessaire développement du front sud. En effet, le groupe réalise ses opérations par le biais de ses katibas dans un territoire extrêmement étendu. Ses structures de mobilisation ne permettent une centralisation du pouvoir à travers les organes de décision du groupe. Les katibas, constamment en mouvement, épousant une réalité nomade, se doivent de s'adapter continuellement aux contraintes locales et ne peuvent donc que s'autonomiser et échapper au contrôle de l'émir d'AQMI. La difficulté de centraliser le pouvoir est ainsi inhérente à la nature même du groupe, modelée par les contextes structurels et les contraintes et opportunités en découlant. Le groupe tend donc à se structurer davantage en un réseau subdivisé en sous-groupes autonomes.

Toutefois, l'on ne peut ôter toute idée d'une structure organisationnelle hiérarchique dans le cas d'AQMI. L'assemblée du Conseil des chefs, tenue en septembre 2008, à la suite d'une opposition entre Belmokhtar et Abou Zaïd sur les sources de financement du groupe, l'illustre parfaitement. En effet, au cours de cette assemblée, le Conseil des chefs donne raison à Abou Zaïd en priorisant le financement par les enlèvements d'étrangers (Guidère, 2011). Le financement par les activités de contrebande est par ailleurs interdit, contraignant de ce fait davantage Belmokhtar, tout en le rendant moins nécessaire au financement du groupe. Les « institutions » du groupe ont donc une réalité, mais ne peuvent encadrer l'ensemble des actions des émirs sahéliens.

Au cours des dernières années, Belmokhtar et Abou Zaïd se sont d'ailleurs livrés à une véritable course à l'enlèvement d'Occidentaux et ont accru sensiblement la visibilité du front sud d'AQMI et son développement. Le nord du Mali constitue le sanctuaire du groupe, où la plupart du temps les otages sont négociés et libérés (ICG, 2012). Du reste, les négociateurs, souvent membres des tribus locales, entretiennent des relations ambiguës avec les membres d'AQMI (ICG, 2012).

Le développement d'AQMI dans la région sahélo-saharienne est ainsi à la fois le résultat de choix stratégiques pris en réponse aux contraintes et opportunités vécues par le groupe, mais aussi le résultat des dynamiques internes du groupe résumées dans les relations de pouvoir changeantes des décideurs et les différentes restructurations organisationnelles.

Par ailleurs, l'autonomisation progressive des katibas du front sud, conforté par l'isolement et l'affaiblissement de Droukdal au nord, conduit les différents émirs à développer leurs propres agendas politiques, renforçant un climat de tensions à l'instar des relations entre Belmokhtar et Abou Zaïd. De plus, les revendications des membres sahéliens se font de plus en plus importantes, car la réalité portée par AQMI ne peut s'empêcher d'évoluer en devenant « sahélienne ». Ainsi, la création du Mouvement pour l'unicité et le jihad en Afrique de l'Ouest (MUJAO) est survenue à la suite d'une défection de membres d'AQMI. Ces derniers souhaitaient la constitution d'une katiba dirigée par un Maure du Sahel (Lounnas, 2012). Le refus des émirs d'AQMI de constituer cette katiba a donc conduit à l'établissement de cette organisation dissidente. L'extension du théâtre d'opérations conduit le groupe à acquérir une certaine lâcheté dans la structure organisationnelle, mais aussi à de nécessaires prises en compte des membres sahéliens au sein de la structure pour éviter des tensions et des dissidences. La constitution du MUJAO, engendré par ces dissidences, rend compte du challenge vécu par AQMI dans son adaptation constante aux contextes structurels et aux restructurations induites à l'échelle du groupe.

Néanmoins, lors de l'insurrection amorcée en 2012 au nord du Mali, AQMI, s'inscrivant dans une dynamique de survie et souhaitant conserver son sanctuaire sahélien, s'est fait l'alliée du groupe islamiste Ansar Eddine et du MUJAO (Lounnas, 2012; ICG, 2012). Répétant à de nombreuses reprises ses menaces d'exécution des otages français en cas d'une intervention militaire de la France au Mali, AQMI semblait craindre plus que jamais la perte de son sanctuaire et l'affrontement armé. Par ailleurs, à la mort de Nabil Makhloufi, numéro deux d'AQMI, en septembre 2012, le groupe nomme Yahia Abou El-Hammam pour le 
remplacer. Ce choix consensuel, car convenant à Droukdal et à tous les émirs sahéliens est le reflet d'une volonté du groupe de rester unie au nom de l'impératif de survie.

Toutefois, la marginalisation progressive de Belmokhtar et les tensions exacerbées avec Droukdal au cours de l'insurrection nord-malienne conduisent à sa défection. Belmokhtar quitte AQMI finalement en décembre 2012 pour créer son propre groupe : " les Signataires par le sang ». Les nombreuses restructurations organisationnelles du groupe et les rapports de force changeants ont entraîné cette dissidence facilitée et accélérée par un contexte structurel s'y prêtant. Cette dernière dissidence est le résultat de dynamiques internes et s'explique par l'autonomisation du front sud, la marginalisation progressive de Belmokhtar dans la hiérarchie d'AQMI ainsi que la volonté de limiter ses activités dans le cadre du groupe par Droukdal. Par ailleurs, le contexte structurel, faisant vivre un stress important au groupe, les divergences entre les émirs dans la manière d'appréhender la survie du front sud ont vraisemblablement amené à ce point de rupture.

Le groupe s'est inscrit tout au long de son histoire dans une dynamique de survie, mais les contextes structurels ont conduit à l'élargissement du théâtre d'opérations, amenant à une autonomisation progressive des structures de mobilisation que sont les katibas. Les rapports de force au sein du groupe, évoluant aussi en fonction des contextes structurels et des restructurations de l'organisation, conduisent nécessairement à constater qu'AQMI est vulnérable aux divisions internes et aux dissidences. Son sanctuaire étant menacé au nord du Mali par l'intervention des forces françaises et africaines, AQMI est vraisemblablement appelée à changer de théâtre d'opérations et à évoluer suite à de nouvelles dynamiques internes.

\section{Conclusion}

Les théories des mouvements sociaux présentent l'avantage d'offrir une analyse des groupes jihadistes sur plusieurs niveaux. En mobilisant les niveaux macro- et méso-analytiques et en les mettant en dialectique, les différentes étapes du développement d'AQMI dans la région sahélo-saharienne ont pu être mises en perspective dans la présente étude. Le développement du groupe dans la région sahélo-saharienne est le résultat des choix stratégiques pris par le groupe en réponse aux différents contextes structurels, c'est-àdire aux contraintes et opportunités externes. Néanmoins, il est aussi le fruit des dynamiques internes du groupe qui ont abouti parfois à des décisions communes, d'autres fois à des décisions antagonistes, sur fond de tensions. Les contextes structurels ont toutefois certainement favorisé des dynamiques internes, modifiant la structure organisationnelle et les relations de pouvoir au sein d'AQMI.

Ainsi, les choix stratégiques du groupe ne peuvent être dissociés des dynamiques internes et ne peuvent être appréhendés comme étant le reflet de décisions prises par un acteur unitaire rationnel. Les choix stratégiques réalisés par le groupe sont en effet parfois pris collégialement dans le cadre des institutions établies par le groupe et en réaction aux opportunités et contraintes régionales, mais sont aussi influencés par la structure organisationnelle et les relations de pouvoir. Considérer les évolutions d'AQMI au regard de choix stratégiques du groupe pris comme un ensemble cohérent relève donc d'un excès de simplification. Ce biais s'avère être celui des études focalisées sur le niveau macro-analytique. Les dynamiques internes n'expliquent pas à elles seules non plus les évolutions de ce groupe jihadiste. Les contraintes et opportunités régionales ont effectivement autant à jouer que les dynamiques internes dans l'évolution du groupe et s'influencent réciproquement. La dialectique entre les différents niveaux d'analyse est dès lors un outil de compréhension que nous invitons à explorer pour se pencher sur les processus de transformations des groupes jihadistes. L'impératif de survie, auquel souscrit AQMI, nous a fait néanmoins négliger dans la présente étude le niveau micro-analytique. Il convient dans une étude future de l'intégrer aux deux précédents niveaux pour renforcer notre compréhension du groupe.

À propos de l'auteur : Adib Bencherif est détenteur d'une maîtrise en relations internationales des HEI de l'Université Laval. Il est chercheur en résidence à l'Observatoire sur le Moyen-Orient et l'Afrique du Nord de la Chaire Raoul-Dandurand de l'Université du Québec à Montréal. Il peut être contacté à l'adresse courriel suivante : adib.bencherif@gmail.com. 


\section{Références}

Africa center for the study and research on terrorism. (2010). African Journal for the Prevention and Combating of Terrorism, Alger: CAERT.

Beck, C. J. (2008). The contribution of social movement theory to understanding terrorism. Sociology Compass, 2(5), 1565-1581.

Bosi, L. (2012). État des savoirs et pistes de recherche sur la violence politique. Critique internationale, 1(54), 171-189.

Botha, A. (2008). Terrorism in the Maghreb, The Transnationalisation of Domestic Terrorism. ISS Monograph series, 144.

Daniel, S. (2012). AQMI. L'industrie de l'enlèvement, Paris: Fayard.

Della Porta, D. (1995). Social movements, political violence, and the state: A comparative analysis of Italy and Germany. Cambridge: Cambridge University Press.

Della Porta, D. (2008). Research on social movements and political violence. Qualitative sociology, 31(3), 221-230.

Della Porta, D., \& LaFree, G. (2012). Guest Editorial: Processes of Radicalization and DeRadicalization. International Journal of Conflict and Violence, 6(1), 4-10.

Durand, G. (2011). L'organisation d'Al-Qaïda au Maghreb islamique. Réalité ou manipulations ?, Paris: L'Harmattan.

Duyvestyen, I. (2004). How new is the new terrorism?. Studies in Conflict and Terrorism, 27(5), 439-454.

Filiu, J. P. (2009). The local and global jihad of al-Qa'ida in the Islamic Maghrib. Middle East Journal, $63(2)$.

Filiu, J. P. (2010). Could Al-Qaeda Turn African in the Sahel?. CARNEGIE papers, 112.

Filiu, J. P. (2011). La campagne anti-française d'Al-Qaïda au Sahel. Politique internationale, 131.

Gamson, W. A., \& Meyer, D. S. (1996). Framing political opportunity. Comparative perspectives on social movements: Political opportunities, mobilizing structures, and cultural framings, (275-290). Cambridge: Cambridge University Press.

Guidère, M. (2008). La tentation internationale d'Al-Qaïda au Maghreb. IFRI, Focus stratégique, 12.

Guidère, M. (2011). Al-Qaïda au Maghreb islamique: le tournant des révolutions arabes, MaghrebMachrek, 208, 59-73.

Hafez, M. M. (2004). From Marginalization to Massacres, A Political Process Explanation of GIA Violence in Algeria. In Q. Wiktorowicz (Eds.), Islamic Activism: A Social Movement Theory Approach (pp.37-60). Bloomington, IN: Indiana University Press.

International Crisis Group. (2005). Islamist Terrorism in the Sahel: Fact or Fiction?, (Crisis Group Africa Report No.92).

International Crisis Group. (2012). Mali, éviter l'escalade, (Rapport d'Afrique No.189).

Lounnas, D. (2011). AQMI, filiale d'Al-Qaida ou organisation algérienne?. Maghreb-Machrek, 208, 3757.

Lounnas, D. (2012). Al-Qaïda au Maghreb islamique et la crise malienne. Sécurité globale, 21, 41-56.

Marret, J. L. (2008), Al-Qaeda in Islamic Maghreb: A "Glocal” Organization. Studies in Conflict \& Terrorism, 31(6), 541-552.

McAdam, D., McCarthy, J. D., \& Zald, M. N. (Eds.). (1996). Opportunities, mobilizing structures, and framing processes- toward a synthetic, comparative perspective on social movements, Comparative perspectives on social movements: Political opportunities, mobilizing structures, and cultural framings (1-20). Cambridge: Cambridge University Press.

McAdam, D. (1996). Conceptual origins, current problems, future directions. Comparative perspectives on social movements: Political opportunities, mobilizing structures, and cultural framings (23-40). Cambridge: Cambridge University Press.

Oberschall, A. (2004). Explaining terrorism: The contribution of collective action theory. Sociological Theory, 22(1), 26-37. 
Pham, J. P. (2011). The Dangerous "Pragmatism" of Al-Qaeda in the Islamic Maghreb. The Journal of the Middle East and Africa, 2(1), 15-29.

Porter, G. (2011). AQIM'S objectives in North Africa. CTC Sentinel, 4(2), 5-8.

Rapin, A. J. (2008). L'objet évanescent d'une théorie improbable: le terrorisme et les sciences sociales. Les Cahiers du RMES, 5(1), 165-213.

Revillard, A. (2003). La sociologie des mouvements sociaux : Structures de mobilisations, opportunités politiques et processus de cadrage. Retrieved from http://annerevillard.files.wordpress.com/2012/01/revillard-2003-socio-des-ms-doc-de-travail.pdf.

Rucht, D. (1996). The impact of national contexts on social movement structures: A cross-movement and cross-national comparison. Comparative perspectives on social movements: Political opportunities, mobilizing structures, and cultural framings, (185-204). Cambridge: Cambridge University Press.

Sidel J. T. (2006). Riots, Progroms, Jihad: Religious Violence in Indonesia. Ithaca: Cornell University Press.

Smith, G. A. (2009) Al-Qaeda in the Lands of the Islamic Maghreb. Journal of Strategic Studies, 2(2), 5372.

Snow, D. A., Rochford, E. B., Worden, S., \& Benford, R. D., (1986). Frame alignment processes, micromobilization, and movement participation. American Sociology Review, 51 (4), 464-481.

Walther, O., \& Retaille, D. (2011). Guerre au Sahara-Sahel : la reconversion des savoirs nomades. L'information géographique, 75(3), 51-68.

Wiktorowicz, Q. (2004). Islamic Activism and Social Movement Theory. In Q.Wiktorowicz (Eds.), Islamic Activism: A Social Movement Theory Approach (pp.1-33). Bloomington, IN: Indiana University Press. 